\title{
Ketone supplementation decreases tumor cell viability and prolongs survival of mice with metastatic cancer
}

\author{
A.M. Poff ${ }^{1}$, C. Ari $^{1}$, P. Arnold ${ }^{2}$, T.N. Seyfried ${ }^{3}$ and D.P. D’Agostino ${ }^{1}$ \\ ${ }^{1}$ Department of Molecular Pharmacology and Physiology, Morsani College of Medicine, Hyperbaric Biomedical Research Laboratory, University of \\ South Florida, Tampa, FL \\ ${ }^{2}$ Savind, Inc., Seymour, IL \\ ${ }^{3}$ Department of Biology, Boston College, Chestnut Hill, MA
}

Cancer cells express an abnormal metabolism characterized by increased glucose consumption owing to genetic mutations and mitochondrial dysfunction. Previous studies indicate that unlike healthy tissues, cancer cells are unable to effectively use ketone bodies for energy. Furthermore, ketones inhibit the proliferation and viability of cultured tumor cells. As the Warburg effect is especially prominent in metastatic cells, we hypothesized that dietary ketone supplementation would inhibit metastatic cancer progression in vivo. Proliferation and viability were measured in the highly metastatic VM-M3 cells cultured in the presence and absence of $\beta$-hydroxybutyrate $(\beta \mathrm{HB})$. Adult male inbred VM mice were implanted subcutaneously with firefly luciferase-tagged syngeneic VM-M3 cells. Mice were fed a standard diet supplemented with either 1,3-butanediol (BD) or a ketone ester (KE), which are metabolized to the ketone bodies $\beta \mathrm{HB}$ and acetoacetate. Tumor growth was monitored by in vivo bioluminescent imaging. Survival time, tumor growth rate, blood glucose, blood $\beta \mathrm{HB}$ and body weight were measured throughout the survival study. Ketone supplementation decreased proliferation and viability of the VM-M3 cells grown in vitro, even in the presence of high glucose. Dietary ketone supplementation with BD and KE prolonged survival in VM-M3 mice with systemic metastatic cancer by 51 and $69 \%$, respectively $(p<0.05)$. Ketone administration elicited anticancer effects in vitro and in vivo independent of glucose levels or calorie restriction. The use of supplemental ketone precursors as a cancer treatment should be further investigated in animal models to determine potential for future clinical use.

Key words: Warburg effect, ketone, metastasis

Abbreviations: $\beta \mathrm{HB}$ : beta-hydroxybutyrate; AcAc: acetoacetate; BD: 1,3-butanediol; CR: calorie restriction; FDG-PET: fluorodeoxyglucose positron emission tomography; Glu: glucose; HDAC: histone deacetylase; i.p.: intraperitoneal; $\mathrm{KD}$ : ketogenic diet; KE: ketone ester; MCT: monocarboxylate transporter; mtDNA: mitochondrial DNA; ROS: reactive oxygen species; s.c.: subcutaneous; SCOT: succinyl CoA 3-ketoacid CoA transferase

Conflicts of interest: Patent 12B152PRWO; University of South Florida; D.P. D’Agostino, A. Poff, P. Arnold, "Targeting cancer with metabolic therapy and hyperbaric oxygen.” P. Arnold (Savind Inc.) has received financial support (ONR N000140910244) from D.

D'Agostino (USF) to synthesize ketone esters. The remaining authors have no conflicts of interest

This is an open access article under the terms of the Creative Commons Attribution-NonCommercial-NoDerivs License, which permits use and distribution in any medium, provided the original work is properly cited, the use is non-commercial and no modifications or adaptations are made.

Grant sponsor: Scivation Inc

DOI: $10.1002 / \mathrm{ijc} .28809$

History: Received 16 Sep 2013; Accepted 11 Feb 2014; Online 26 Feb 2014

Correspondence to: Angela M. Poff, 12901 Bruce B. Downs Blvd, MDC 8, Tampa, FL 33612, USA, Fax: +813-974-3079,

E-mail: abennett@health.usf.edu
A century ago, Otto Warburg discovered that cancer cells display a unique metabolic phenotype of lactate fermentation in the presence of oxygen. This phenotype, known as the Warburg effect, enables tumor visualization using fluorodeoxyglucose positron emission tomography (FDG-PET) scans owing to the elevated rate of glucose consumption in most cancers. Metabolic therapies can exploit this phenotype, offering novel therapeutic directions aside from the classically targeted cytotoxic and gene-based therapies. The Warburg effect exposes a fundamental weakness of cancer cells, reliance on excess glucose for survival and maximal proliferation. Fasting, calorie restriction (CR) and the carbohydrate-restricted ketogenic diet have been successfully used to limit glucose availability and slow cancer progression in a variety of animal models and human studies. ${ }^{1-9}$ These dietary manipulations produce a physiological metabolic shift to an unfavorable environment for glucose-dependent cancer cells. Previously, the anticancer effects of these dietary manipulations have largely been attributed to decreased circulating blood glucose, which limits energy substrates for cancer cells. New evidence suggests, however, that the physiological state of ketosis and elevated circulating ketones also have anticancer effects.

Recently, Fine et al. demonstrated that a carbohydraterestricted ketogenic diet inhibited disease progression and promoted partial remission in patients with advanced 


\section{What's new?}

Unlike healthy tissues, cancer cells cannot efficiently process ketone bodies for energy. Ketones also slow the proliferation of tumor cells. These authors speculated that dietary ketones might halt metastasis. They injected metastatic cancer cells into mice, and then fed them a diet supplemented with ketones. The mice receiving the supplements outlived the mice who did not get the ketone-enriched diet, suggesting that this strategy is worth pursuing further.

metastatic cancers from various tissue origins. ${ }^{10}$ On average, the patients did not exhibit a drop in glucose from baseline, suggesting that decreased glucose availability was not the sole or primary cause of efficacy. Interestingly, the study found that the most important factor dictating the patients' response to therapy was the degree of elevated ketosis from baseline. Indeed, a prominent metabolic shift to higher levels of ketosis correlated with reduced disease progression, stable disease or partial regression. A small number of reports have investigated the effects of ketones on cancer growth in vitro. Acetoacetate (AcAc) and $\beta$-hydroxybutyrate $(\beta \mathrm{HB})$ are the two most abundant and physiologically relevant of the three ketone bodies. Acetone is produced as a nonenzymatic byproduct of AcAc metabolism and is rapidly excreted in the lungs. AcAc supplementation inhibited proliferation and ATP production in seven aggressive human colon and breast cancer cell lines but did not affect proliferation in healthy primary fibroblasts. ${ }^{11}$ Similarly, $\beta \mathrm{HB}$ inhibited proliferation of transformed lymphoblasts, HeLa cells and melanoma cells in a dose-dependent manner up to $20 \mathrm{mM}^{6}$ In neuroblastoma cells, $\beta \mathrm{HB}$ and AcAc supplementation decreased viability and increased apoptosis, but had no effect on fibroblasts. ${ }^{12}$

Although ketone bodies are efficient energy substrates for healthy extrahepatic tissues, ${ }^{13}$ cancer cells cannot effectively use them for energy. Widespread mitochondrial pathology has been observed in most if not all tumors examined, including decreased mitochondrial number, abnormal ultrastructural morphology, mitochondrial swelling, abnormal fusion-fission, partial or total cristolysis, mtDNA mutations, altered mitochondrial membrane potential and abnormal mitochondrial enzyme presence or function, among others. ${ }^{14-19}$ These defects in mitochondrial structure and function impair respiratory capacity and force a reliance on substrate-level phosphorylation for survival. ${ }^{20}$ As ketone bodies are metabolized exclusively within the mitochondria, cancer cells with impaired mitochondrial function are unable to efficiently metabolize ketone bodies for energy. Indeed, unlike healthy cells, ketone bodies fail to rescue glioma cells from glucose withdrawal-induced death. ${ }^{21}$

Although mitochondrial dysfunction explains the inability of cancer cells to effectively use ketones for energy, the anticancer effects of ketones in a normal glucose in vitro environment are not immediately clear. Upon closer examination, ketone bodies possess many characteristics that can impair cancer cell survival and proliferation. (i) Ketone bodies inhibit glycolysis, thus decreasing the main pathway of energy production for cancer cells. ${ }^{22}$ (ii) Cancer cells thrive in an environment of elevated reactive oxygen species (ROS) production but are very sensitive to even small changes in redox status. ${ }^{23}$ Ketones decrease mitochondrial ROS production and enhance endogenous antioxidant defenses in normal cells, but not in cancer cells. ${ }^{13}$ Ketone metabolism in healthy cells near the tumor may inhibit cancer cell growth by creating a less favorable redox environment for their survival. (iii) Ketone bodies are transported into the cell through the monocarboxylate transporters (MCTs), which are also responsible for lactate export. It has been shown that inhibiting MCT1 activity or inhibiting lactate export from the cell dramatically decreases cancer cell growth and survival. ${ }^{24}$ Ketones may impair cancer cells indirectly by competitive inhibition of the MCTs, decreasing critical lactate export from the cell. (iv) Recently, Verdin and coworkers demonstrated that $\beta \mathrm{HB}$ acts as an endogenous HDAC inhibitor at millimolar concentrations easily achieved through fasting, CR or ketone supplementation such as with a ketone ester (KE). ${ }^{25}$ Thus, ketone bodies may elicit their anticancer effects by altering the expression of oncogenes and tumor suppressor genes under control of the cancer epigenome. ${ }^{26}$ Clearly, ketone bodies exhibit several unique characteristics that support their use as a metabolic therapy for cancer.

The Warburg effect is especially prevalent in aggressive cancers and metastatic cells. Metastasis, the spreading of a primary tumor to distal locations, is the primary cause of cancer morbidity and mortality and is responsible for more than $90 \%$ of cancerrelated deaths. ${ }^{27}$ To make significant strides toward long-term cancer management, we must evaluate therapies that are effective against late-stage metastatic cancers. The major obstacle preventing the discovery of such treatments has been the lack of animal models that mimic the true metastatic phenotype and accurately predict the clinical success of therapeutics. The VM-M3 model of metastatic cancer is a novel murine model that closely mimics the natural progression of cancer invasion and metastasis. ${ }^{28}$ The original VM-M3 tumor arose as a spontaneous brain tumor in a VM/ Dk inbred mouse. The VM-M3 tumor was adapted to cell culture and transduced with the firefly luciferase gene to allow for in vivo bioluminescent tracking of tumor growth. ${ }^{29} \mathrm{VM}-\mathrm{M} 3$ cells express many in vitro and in vivo characteristics of human glioblastoma multiforme with macrophage or microglial properties. ${ }^{28-30}$ When implanted subcutaneously, the VM-M3 cells are morphologically similar to histiocytes and rapidly produce systemic metastasis, forming tumors in all major organ systems, most notably the liver, lung, kidney, spleen, brain and bone. ${ }^{28}$ The VM-M3 model of metastatic cancer spreads naturally in an immunocompetent host, and chemotherapeutic agents inhibit metastatic spread in this model similar to their observed effects in humans. ${ }^{31}$ The 
VM-M3 model has many advantages that support its use as a representation of the true metastatic disease state and has therefore been used in our study.

Although the literature strongly suggests that ketone bodies impede cancer growth in vitro, the in vivo efficacy of dietary ketone supplementation has not been adequately examined. It is possible to raise blood ketone levels without the need for carbohydrate restriction by administering a source of supplemental ketones or ketone precursors. 1,3Butanediol (BD) is a commercially available food additive and hypoglycemic agent that is converted to $\beta \mathrm{HB}$ by the liver. $^{32,33}$ The KE elevates both AcAc and $\beta \mathrm{HB}$ in a dosedependent manner to levels beyond what can be achieved with the KD or therapeutic fasting. ${ }^{34}$ Oral administrations of $\mathrm{BD}$ and $\mathrm{KE}$ have been shown to elevate blood ketones for at least 240 min in rats. ${ }^{34}$ As ketone bodies appear to elicit anticancer effects, and metastasis is the most significant obstacle in the successful treatment of neoplasms, we tested the efficacy of ketone supplementation in the VM-M3 cell line and mouse model of metastatic cancer.

\section{Material and Methods Cell culture}

VM-M3/Fluc cells were received as a gift from T. Seyfried (Boston College) where they were obtained from a spontaneous tumor in a VM/Dk inbred mouse and adapted to cell culture. ${ }^{28}$ VM-M3 cells were transduced with a lentivirus vector containing firefly luciferase under control of the cytomegalovirus promoter (VM-M3/Fluc). ${ }^{28}$ The VM-M3/Fluc cells were cultured in Eagle's minimum essential medium with $2 \mathrm{mM}$ L-glutamine (ATCC, Manassas, VA), 10\% fetal bovine serum (Invitrogen, Grand Island, NY), 1\% penicillin-streptomycin (Gibco, Invitrogen) and high glucose (25 mM D-glucose; Fisher Scientific, Waltham, MA). Cells were maintained at $37^{\circ} \mathrm{C}$ in $95 \%$ air and $5 \% \mathrm{CO}_{2}$.

\section{Proliferation}

VM-M3/Fluc cells were plated into $35-\mathrm{mm}$ six-well plates at a density of 50,000 cells $/ \mathrm{mL}$ in high $(25 \mathrm{mM})$ or low $(3 \mathrm{mM})$ glucose media with or without ketone supplementation (5 mM $\beta \mathrm{HB}$; Sigma-Aldrich, St. Louis, MO). Nonketonesupplemented media contained an equal volume of $\beta \mathrm{HB}$ diluent $\left(50 \mu \mathrm{L} \mathrm{dH_{2 }} \mathrm{O}\right)$. Cells were grown for 24, 48, 72, 96, 120 and $144 \mathrm{hr}$ to determine a growth curve. All media were replaced with respective treatments every $48 \mathrm{hr}$. Each time point was run in triplicate. Cells were collected by scraping and counted using standard trypan blue hemocytometry. Plates were visually inspected to ensure all cells were harvested before counting.

\section{Viability}

Viability was assessed with the LIVE/DEAD® Viability/Cytotoxicity Kit (Invitrogen). VM-M3/Fluc cells were plated into 22-mm 12-well plates containing poly-D-lysine-coated glass coverslips (BrainBits, Springfield, IL) at a density of 50,000/
$\mathrm{mL}$ in control media and allowed to grow for $24 \mathrm{hr}$. At 24 $\mathrm{hr}$, media were replaced with treatment [high $(25 \mathrm{mM})$ or low $(3 \mathrm{mM})$ glucose] with or without $5 \mathrm{mM} \beta \mathrm{HB}$ ketone supplementation, and cells were allowed to grow for $24 \mathrm{hr}$. Nonketone-supplemented media contained an equal volume

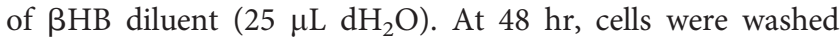
with D-PBS and incubated in $2 \mu \mathrm{M}$ calcein AM (Ex/Em: 495/ $515)$ and $4 \mu \mathrm{M}$ ethidium homodimer-1 (Ex/Em: 525/590) in Dulbecco's phosphate buffered saline (D-PBS) for $30 \mathrm{~min}$ at $37^{\circ} \mathrm{C}$. After incubation, wet coverslips were inverted, mounted and sealed on a microscope slide. Cells were visualized using fluorescence confocal microscopy with FIT-C and TRIT-C filters to identify live and dead cells, respectively. Each treatment was run in triplicate with ten frames analyzed per coverslip with a Nikon TE2000E using a $10 \times$ objective. The numbers of live and dead cells were counted per frame to determine percent viability.

\section{Mice}

Three breeding pairs of the VM/Dk inbred strain of mice were used to establish and propagate a VM/Dk mouse colony in the University of South Florida Morsani College of Medicine Vivarium according to standard murine husbandry protocol. Adult male mice (2-4 months of age) were used for our study, with all procedures completed within strict adherence to the NIH Guide for the Care and Use of Laboratory Animals and were approved by the University of South Florida Institutional Animal Care and Use Committee (IACUC; protocol number R4137).

\section{Tumor implantation}

Approximately 1 million VM-M3/Fluc cells in $300 \mu \mathrm{L}$ PBS were implanted s.c. into the abdomen of VM/Dk mice using a $27 \mathrm{~g}$ needle. VM-M3/Fluc inoculation results in rapid and systemic metastasis as previously described. ${ }^{28}$

\section{Ketone supplementation and diet administration}

Mice were fasted overnight before tumor inoculation and randomly assigned to treatment group. Control mice received standard diet (SD) rodent chow (2018 Teklad Global 18\% Protein Rodent Diet, Harlan) composed of $60 \%$ carbohydrate fed ad libitum. Mice receiving supplemental ketones were administered their respective diet ad libitum in lieu of standard rodent chow. Mice in the BD treatment group received standard rodent chow mixed with $\mathrm{BD}$ at $20 \%$ by volume and $1 \%$ saccharin for palatability (Sigma-Aldrich). Mice in the KE treatment group received standard rodent chow mixed with $\mathrm{KE}$ at $10 \%$ by volume and $1 \%$ saccharin (Sigma-Aldrich) for palatability. The KE was synthesized in collaboration with Savind, Seymour, IL, as previously described. ${ }^{34}$ As ketosis has been shown to decrease appetite and food intake, ${ }^{35}$ and CR is known to inhibit cancer progression, ${ }^{2,36}$ we included a CR treatment group. CR mice received a daily food allotment of standard rodent chow restricted $40 \%$ by weight compared to their normal food consumption. They received their daily 
food allotment at $\sim 3 \mathrm{pm}$. Ad libitum diets were continuously monitored and replaced twice weekly or as needed to maintain freshness.

\section{Blood and weight measurements}

Acute metabolic response to diet in healthy $\mathrm{VM} / \mathrm{dk}$ mice. Blood ketones rise and fall following ingestion of the ketonesupplemented diets, and their levels are affected by the amount of food consumed during a feeding event as well as the time between feeding and blood measurement. Because food was provided ad libitum to mice in the VM-M3 cancer survival study, it was difficult to accurately observe the effects of each diet on blood metabolites. Furthermore, deterioration of health during disease progression affected feeding behavior and, subsequently, blood metabolite concentration. To demonstrate the effects of the diets on blood metabolites without the confounding effects of ad libitum feeding and disease progression, and to demonstrate that oral administration of the ketone supplements given with a standard highcarbohydrate diet is capable of elevating ketones in VM/Dk mice, blood glucose and $\beta \mathrm{HB}$ were measured in a small group of healthy mice following a controlled feeding event. Healthy VM/Dk mice ( $N=4$ per treatment) were fasted for $8 \mathrm{hr}$ to ensure rapid feeding compliance, and then provided $\mathrm{SD}, \mathrm{BD}$ or KE diet ad libitum. All mice fed immediately upon return of food ( $0 \mathrm{hr})$. Blood glucose and $\beta \mathrm{HB}$ were measured at $0,1,4,8$ and $12 \mathrm{hr}$, and urine AcAc was measured at $12 \mathrm{hr}$. Blood was collected from the tail using approved methods, and glucose and $\beta \mathrm{HB}$ were measured using the Precision Xtra ${ }^{\mathrm{TM}}$ Blood Glucose \& Ketone Monitoring System (Abbott Laboratories, Abbott Park, IL). Urine AcAc was measured using Ketocare Reagent Strips for Urinalysis (CVS, Woonsocket, RI).

VM-M3 cancer survival study blood and weight measurements. Blood was collected once weekly from the tail of the VM-M3 cancer mice using approved methods. Blood glucose was measured with the Nova Max® Plus Glucose and $\beta$-Ketone Monitoring System (Nova Biomedical, Waltham, $\mathrm{MA}$ ), and blood $\beta \mathrm{HB}$ was measured with the Precision Xtra $^{\mathrm{TM}}$ Blood Glucose \& Ketone Monitoring System (Abbott Laboratories). Mice were weighed between 1 and $3 \mathrm{pm}$, twice weekly, for the duration of the study using the AWS-1KG Portable Digital Scale (AWS, Charleston, SC).

\section{Bioluminescent imaging and tumor growth analysis}

Tumor growth and metastatic spread were monitored by in vivo bioluminescent imaging with the Xenogen IVIS Lumina system (Caliper LS, Hopkinton, MA). Bioluminescent signal of the luciferase-tagged cancer was acquired with the Living Image ${ }^{\circledR}$ software (Caliper LS). Mice received an i.p. injection of $50 \mathrm{mg} / \mathrm{kg}$ D-luciferin (Caliper LS) $15 \mathrm{~min}$ before imaging. Bioluminescent signal was obtained using the IVIS Lumina cooled CCD camera system with a 1-sec exposure time. Whole animal bioluminescent signal was measured in pho- tons/sec once a week as an indicator of overall tumor burden. Bioluminescent signal of key metastatic regions (brain, lungs and liver) was measured in photons/sec as an indicator of metastatic tumor size and spread. ${ }^{37,38}$

\section{Survival analysis}

Health and behavior of the mice were assessed daily for the duration of the study. Upon presentation of defined criteria associated with tumor burden and disease progression (abnormal feeding behavior, diminished response to stimuli and failure to thrive), mice were humanely euthanized according to approved IACUC guidelines and survival time was recorded.

\section{Statistics}

Survival was analyzed with the Kaplan-Meier and log-rank tests for survival distribution. Cell viability was analyzed by unpaired $t$-test. Cell proliferation, whole animal bioluminescent signal, mean survival, weekly blood measurements, percent body weight change and urine ketones were analyzed by one-way analysis of variance (ANOVA) with Tukey's multiple comparison post hoc test. Acute blood measurements were analyzed by two-way ANOVA with Tukey's multiple comparison post hoc test. Correlation between blood glucose, body weight change and survival times was analyzed by linear regression analysis. Results were considered significant when $p<0.05$.

\section{Results \\ Ketone supplementation decreased VM-M3 cell proliferation and viability in vitro}

Proliferation was significantly decreased in VM-M3 cells grown in low $(3 \mathrm{mM})$ glucose conditions and in both low (3 $\mathrm{mM})$ and high $(25 \mathrm{mM})$ glucose conditions with ketone supplementation ( $5 \mathrm{mM} \beta \mathrm{HB}$ ) compared to VM-M3 cells grown in control media (high glucose, $25 \mathrm{mM}$ ) at 24, 48, 72, 96, 120 and $144 \mathrm{hr}(p<0.05$; one-way ANOVA; Fig. 1a). Although low $(3 \mathrm{mM})$ glucose with ketone supplementation $(5 \mathrm{mM} \beta \mathrm{HB})$ demonstrated a trend of decreased proliferation compared to low $(3 \mathrm{mM})$ glucose alone at all time points, the difference was not statistically significant (one-way ANOVA; Fig. 1a). Viability was significantly decreased in VM-M3 cells treated for $24 \mathrm{hr}$ with low $(3 \mathrm{mM})$ glucose conditions and in both low $(3 \mathrm{mM})$ and high $(25 \mathrm{mM})$ glucose conditions with ketone-supplemented ( $5 \mathrm{mM} \beta \mathrm{HB}$ ) media compared to VMM3 cells grown in control media (high glucose, $25 \mathrm{mM}$; $p<0.05$; unpaired $t$-test; Fig. $1 b$ ).

\section{Ketone supplementation prolonged survival and induced a trend of reduced tumor burden in mice with metastatic cancer}

Bioluminescence of tumor burden varied within and between groups according to disease progression and the health and number of animals remaining in each group over time (Fig. 2b). CR-, BD- and KE-treated mice demonstrated a trend of reduced tumor burden (Figs. $2 a$ and $2 b$ ) and 


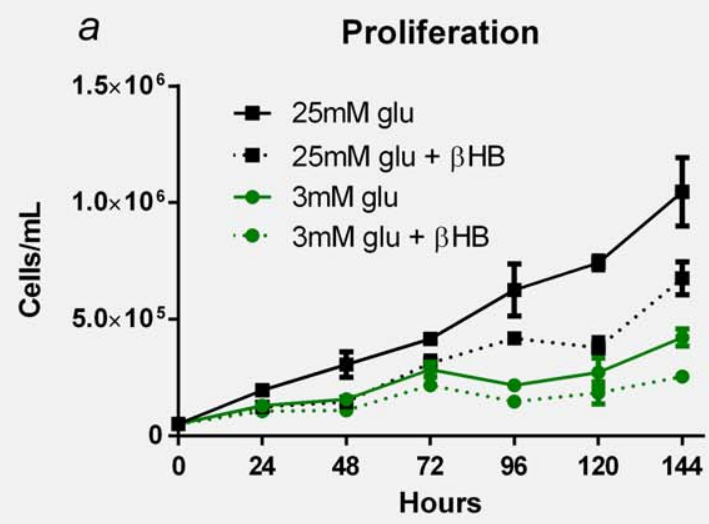

$-\beta \mathrm{HB}$
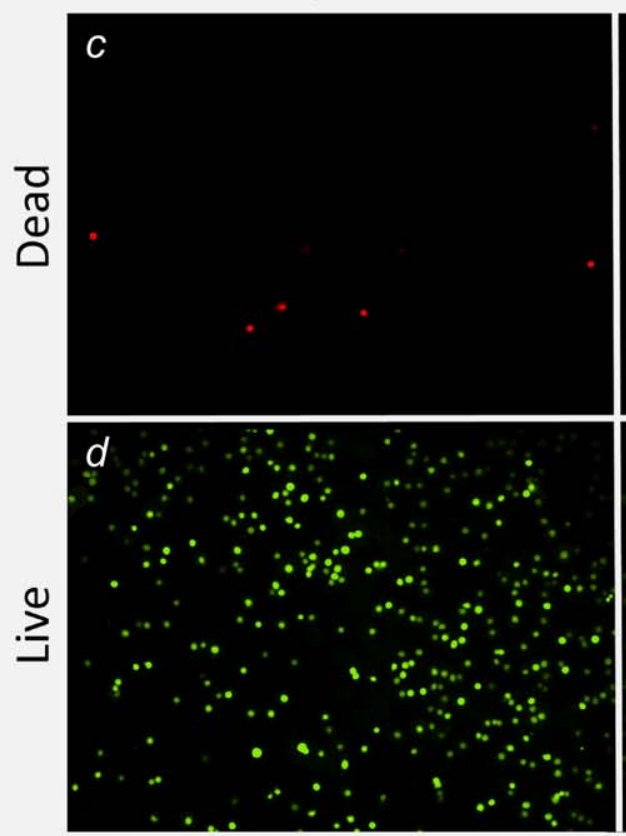

$b$

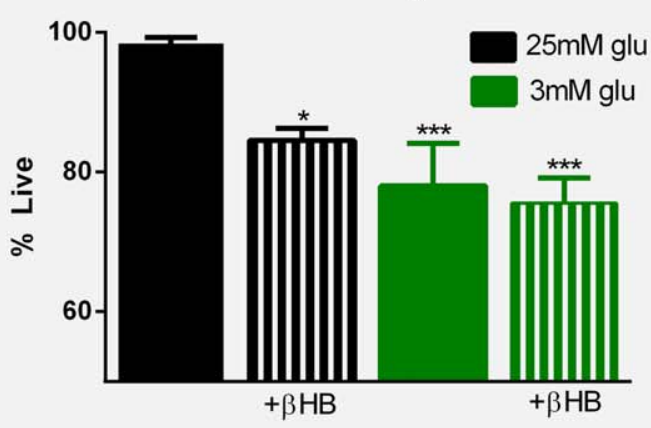

$+\beta \mathrm{HB}$
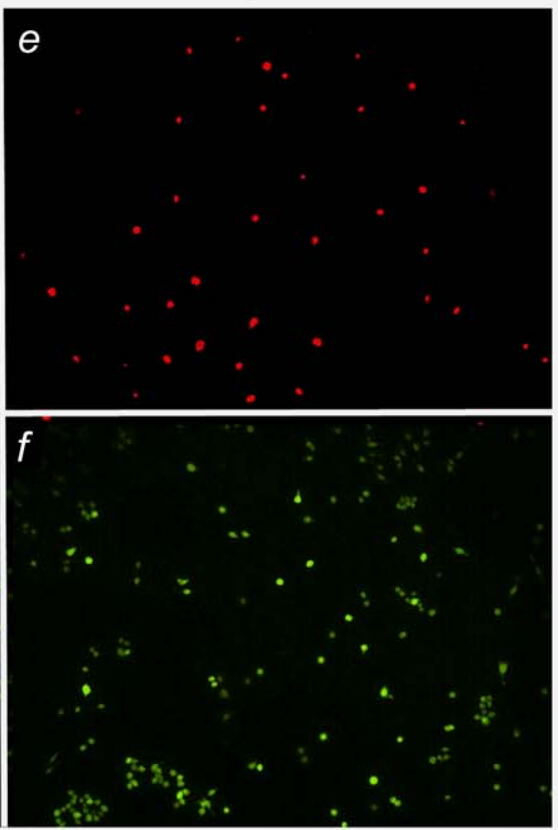

Figure 1. $\beta$ HB decreases VM-M3 cell proliferation and viability in vitro. (a) VM-M3 proliferation was inhibited when grown in low (3 mM) glucose- and/or ketone-supplemented ( $5 \mathrm{mM} \beta \mathrm{HB}$ ) media (Glu: glucose; $\beta \mathrm{HB}$ : $\beta$-hydroxybutyrate). Cell density was significantly less in low (3 $\mathrm{mM})$ glucose conditions and in both low $(3 \mathrm{mM})$ and high $(25 \mathrm{mM})$ glucose conditions with ketone supplementation $(5 \mathrm{mM} \beta \mathrm{HB})$ at 24,48 , 72, 96, 120 and $144 \mathrm{hr}$ compared to high glucose controls ( $p<0.05$; one-way ANOVA). Although low ( $3 \mathrm{mM})$ glucose with ketone supplementation $(5 \mathrm{mM} \beta \mathrm{HB})$ demonstrated a trend of decreased proliferation compared to low $(3 \mathrm{mM})$ glucose alone, the difference was not statistically significant. (b) VM-M3 viability was decreased when grown in low (3 mM) glucose conditions and in both low (3 mM) and high (25 $\mathrm{mM}$ ) glucose conditions with ketone supplementation ( $5 \mathrm{mM} \mathrm{\beta HB}$ ). There was a significantly smaller percentage of live cells in all treated versus control (high glucose, $25 \mathrm{mM})$ media $\left({ }^{\star} p<0.05\right.$, ${ }^{\star \star \star} p<0.001$; unpaired $t$-test). ( $\left.c-f\right)$ Live/dead fluorescence of VM-M3 cells grown in high $(25 \mathrm{mM})$ glucose with and without $5 \mathrm{mM} \beta \mathrm{HB}$. Results were considered significant when $p<0.05$. Error bars represent \pm SEM. [Color figure can be viewed in the online issue, which is available at wileyonlinelibrary.com.]

metastatic spread (Figs. $2 c$ and $2 d$ ) over the course of the survival study; however, bioluminescence was highly variable and not significantly different from controls.

BD- and KE-treated mice demonstrated a significantly prolonged survival curve by log-rank test for survival distribution compared to control animals $(p=0.01$ and $p=0.01$, respectively, Fig. $3 a$ ). They also showed a significant increase in mean survival time compared to control animals (one-way ANOVA; $p<0.05$ and $p<0.001$, respectively; Fig. $3 b$ ).
Although previous reports have demonstrated that CR increases survival time in various animal cancer models, in our study, CR-treated mice exhibited a trend of increased latency to disease progression measured as tumor bioluminescence, but survival time was not statistically different from controls (log-rank test for survival distribution and one-way ANOVA; $p>0.05$; Figs. 2 and 3). Control (SD) mice lived an average of 31.2 days, whereas CR mice had a nonstatistically significant different mean survival time of 36.9 days 
a

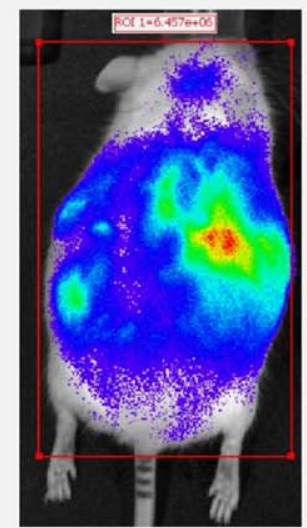

Control (SD)

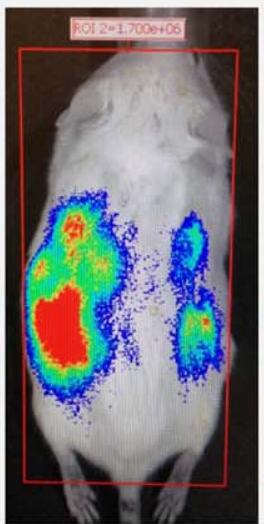

CR

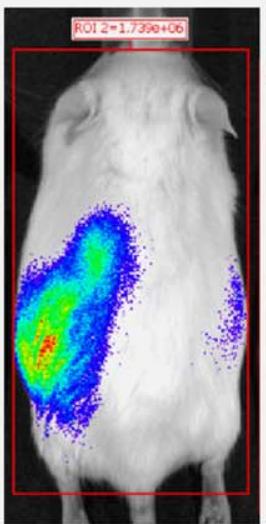

BD

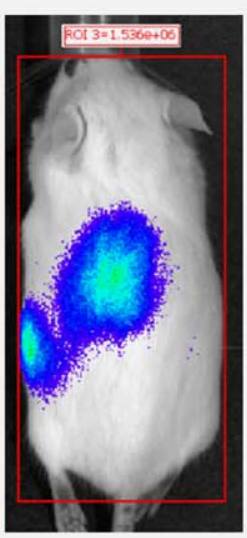

KE Bioluminescence (Photons/sec)

b

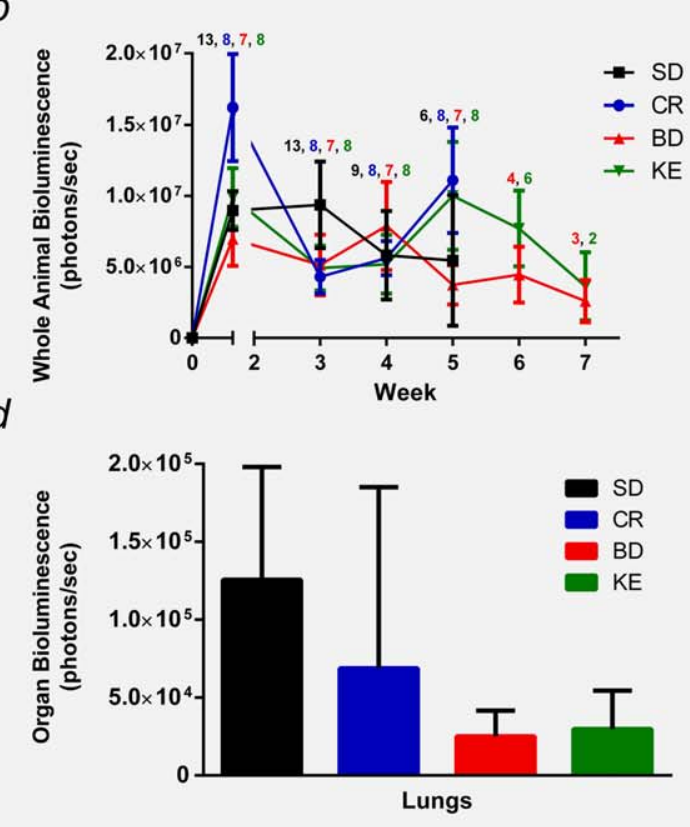

C

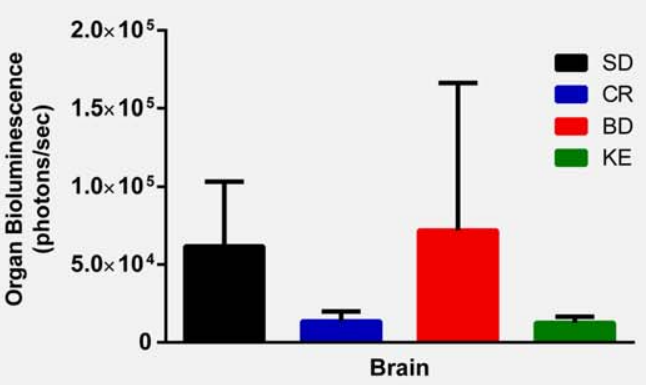

e

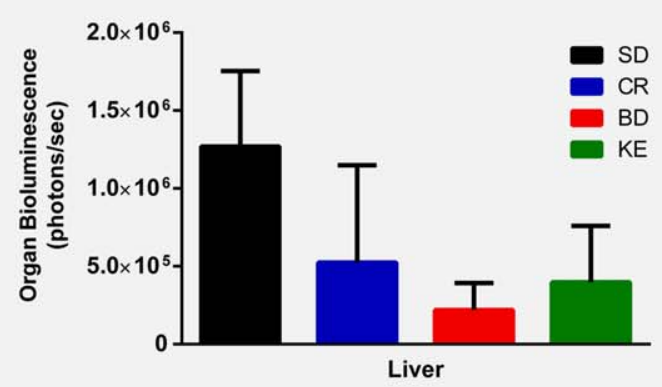

Figure 2. Effect of supplemental ketones on tumor bioluminescence. Ketone supplement-fed mice demonstrated a trend of slower tumor growth and metastatic spread compared to controls. (a) Representative animals from each group at 21 days post-tumor cell inoculation showing whole body tumor bioluminescence. Treated mice exhibited reduced tumor burden and metastatic spread. $(b)$ Whole animal bioluminescence tracked over time as a measure of tumor growth rate. The number of animals alive at each week is presented as the color-coded number above the average bioluminescence data points. ( $c-e$ ) Bioluminescence of key metastatic regions: brain, lungs and liver. Treated animals exhibited a trend of slower tumor growth rate and decreased metastatic spread compared to controls, although bioluminescence was not significantly different from controls. Error bars represent \pm SEM. [Color figure can be viewed in the online issue, which is available at wileyonlinelibrary.com.]

( $p>0.05$; one-way ANOVA; Fig. 3). BD treatment increased mean survival time by $\sim 16$ days $(51 \%)$, and $\mathrm{KE}$ treatment increased mean survival time by $\sim 22$ days (69\%) compared to controls $(p<0.01$ and $p<0.001$, respectively; one-way ANOVA; Fig. $3 b)$.

\section{Ketone supplementation lowered blood glucose, elevated blood ketones and decreased body weight}

Acute ketosis with supplementation in healthy VM/Dk mice. Standard high-carbohydrate rodent chow with ketone supplementation significantly lowered blood glucose for 8 and $4 \mathrm{hr}$ in
$\mathrm{BD}$ and KE groups, respectively ( $p<0.05$; two-way ANOVA). $\mathrm{BD}$ significantly elevated $\beta \mathrm{HB}$ levels after $1 \mathrm{hr}$, which further increased in the next $11 \mathrm{hr}$, whereas $\mathrm{KE}$ caused significant $\beta \mathrm{HB}$ elevation only after $4 \mathrm{hr}$ and remained at a similar level after 12 hr $(p<0.05$; two-way ANOVA). BD elevated only $\beta \mathrm{HB}$, whereas $\mathrm{KE}$ elevated both $\beta \mathrm{HB}$ and AcAc $(p<0.05$; two-way ANOVA and one-way ANOVA, respectively; Figs. $4 b$ and $4 c$ ).

Blood and weight measures in VM-M3 cancer survival study mice. Initial blood glucose, $\beta \mathrm{HB}$ and body weights were similar between groups (data not shown). Chronic ketone 


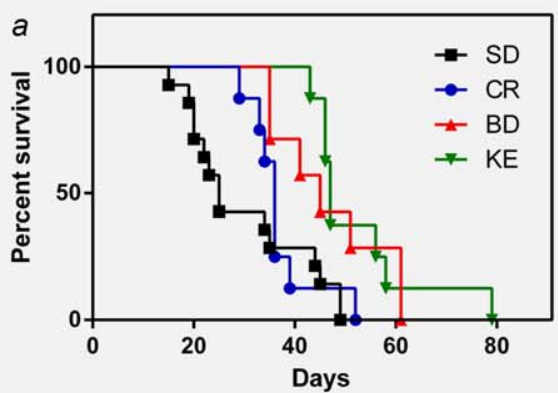

\begin{tabular}{|l|c|c|c|}
\hline & $\begin{array}{c}\text { Mean } \\
\text { Cohort } \\
\text { Tize (N) }\end{array}$ & $\begin{array}{c}\text { Increase in } \\
\text { Survival } \\
\text { Time (days) }\end{array}$ & $\begin{array}{c}\text { Mean Survival } \\
\text { Time }\end{array}$ \\
\hline Control (SD) & 13 & 31.2 & -- \\
\hline CR & 8 & 36.9 & $18.7 \%$ \\
\hline BD & 7 & 47.0 & $50.6 \% *$ \\
\hline KE & 8 & 52.8 & $69.2 \% * * *$ \\
\hline
\end{tabular}

Figure 3. Supplemental ketones extend survival time in mice with systemic metastatic cancer. (a) Kaplan-Meier survival curve of treatment groups. BD- and KE-, but not CR-, treated mice demonstrated prolonged survival compared to controls (log-rank test for survival distribution; $p=0.01, p=0.001$ and $p=0.30$, respectively). (b) Cohort size, mean survival and percent change from mean survival of controls. BD mice exhibited a $51 \%$ increase in mean survival time compared to controls, whereas KE mice exhibited a $69 \%$ increase in mean survival time compared to controls ( ${ }^{*} p<0.05$; ${ }^{* \star *} p<0.001$; one-way ANOVA). Results were considered significant when $p<0.05$. [Color figure can be viewed in the online issue, which is available at wileyonlinelibrary.com.]
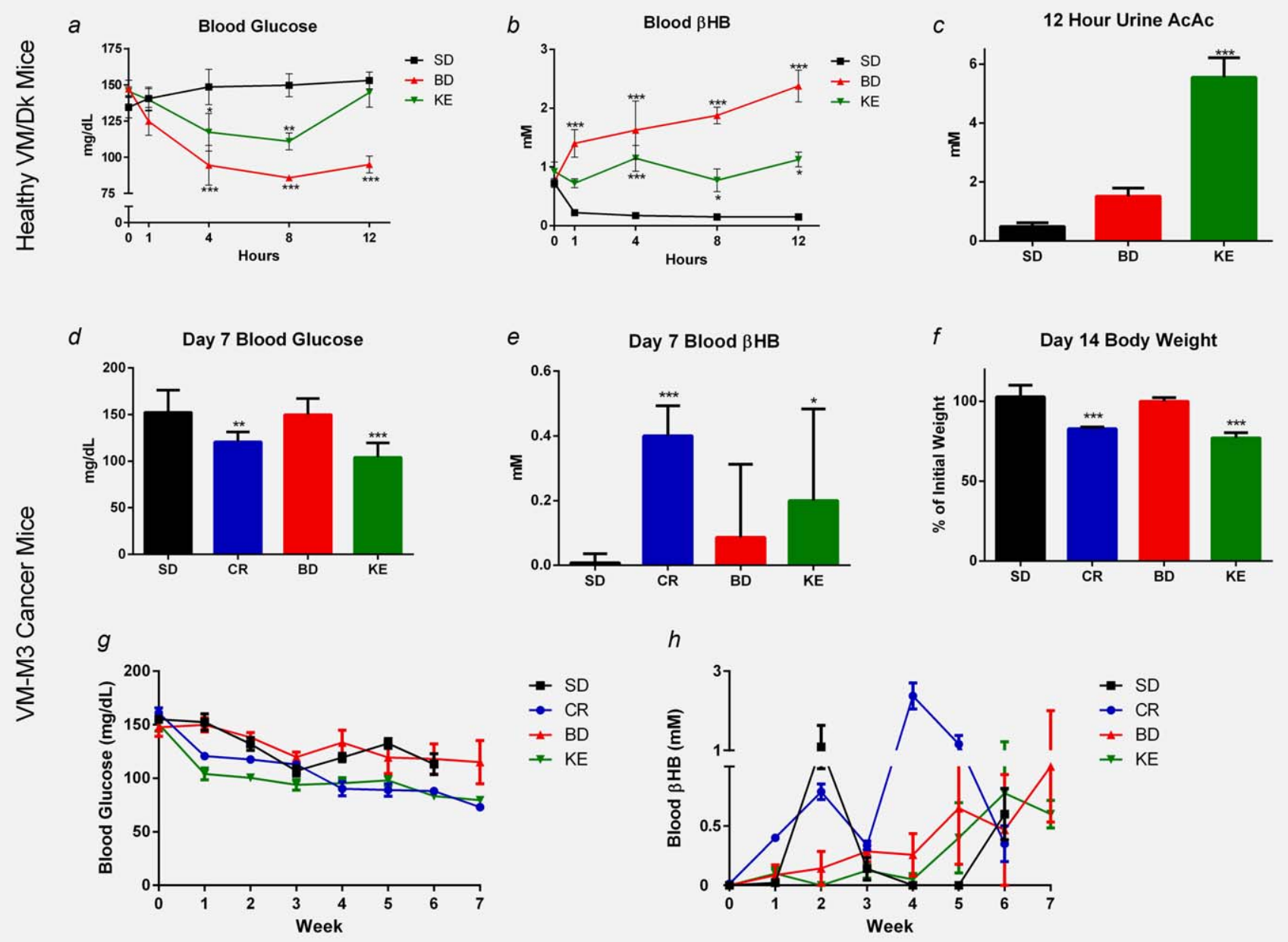

Day 7 Blood $\beta$ HB

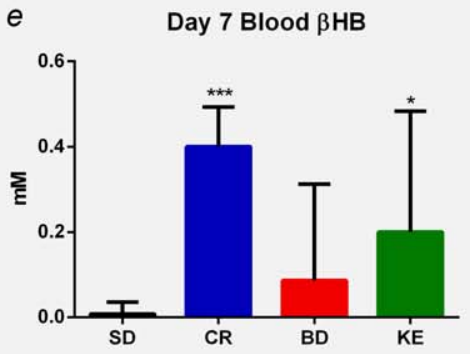

$f$

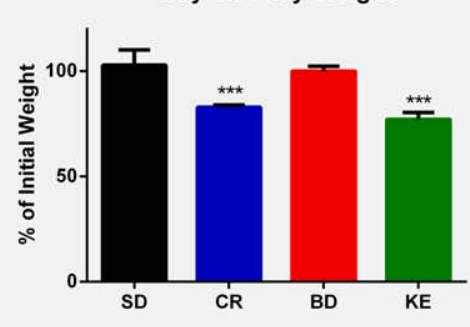

Figure 4. Effect of ketone supplementation on blood glucose, blood $\beta H B$, urine AcAc and body weight in mice. ( $a-c$ ) Glucose and ketones of healthy VM/Dk mice $0-12 \mathrm{hr}$ after feeding following $8 \mathrm{hr}$ fast. BD- and KE-treated mice demonstrated decreased blood glucose and elevated blood $\beta$ HB following feeding ( ${ }^{\star} p<0.01$; ${ }^{* \star} p<0.05$; ${ }^{* \star} p<0.001$; two-way ANOVA). KE-, but not BD-, treated mice demonstrated elevated urine AcAc $12 \mathrm{hr}$ after feeding ( ${ }^{\star \star \star} p<0.001$; one-way ANOVA). ( $d-f$ ) Blood glucose, ketones and body weight of survival study VM-M3 mice before significant disease onset. (d) CR- and KE-treated mice had lower glucose than controls by day 7 (** $p<0.01$; ${ }^{* * *} p<0.001$; one-way ANOVA). (e) CR and KE mice had elevated blood $\beta \mathrm{HB}$ compared to controls at day $7\left({ }^{\star} p<0.05 ;{ }^{* \star} p<0.001\right.$; one-way ANOVA). (f) CR and KE mice had a $20 \%$ reduction in body weight compared to controls at day 14 , which was sustained for the duration of study $(* \star \star p<0.001$; oneway ANOVA). Results were considered significant when $p<0.05$. Error bars represent \pm SEM. $(g$ and $h$ ) Blood glucose and ketones of VM-M3 cancer mice over the course of the survival study. [Color figure can be viewed in the online issue, which is available at wileyonlinelibrary.com.] 

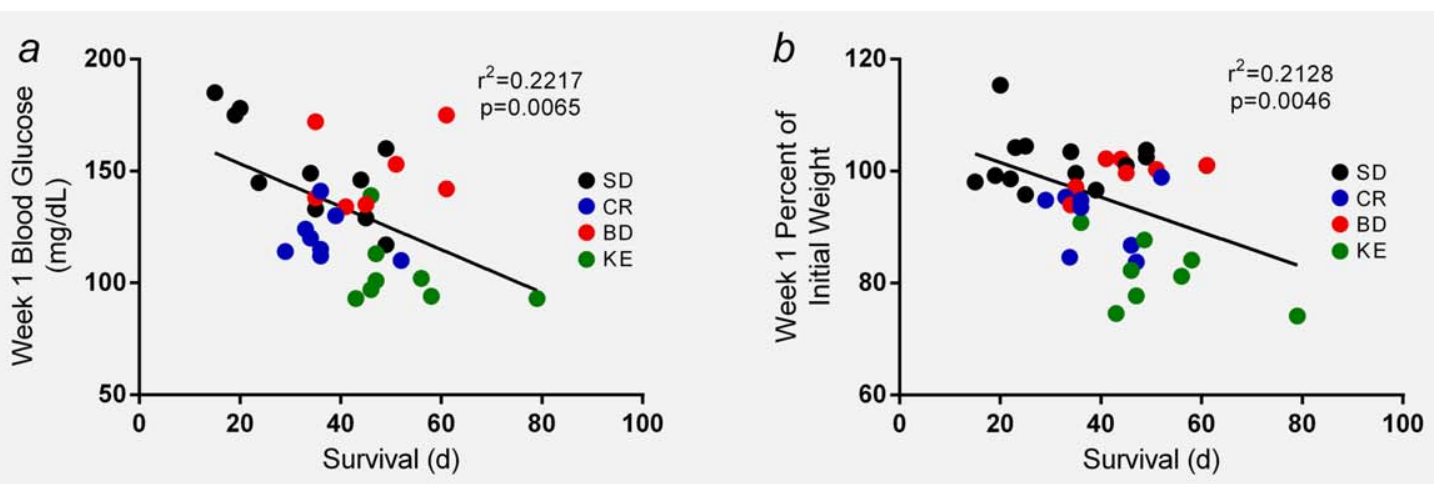

Figure 5. Decreased blood glucose and weight loss correlated with longer survival. Linear regression analysis of day 7 blood glucose and percent body weight change for all study animals revealed a significant correlation to survival time ( $p=0.0065$ and $p=0.0046$, respectively). Results were considered significant when $p<0.05$. [Color figure can be viewed in the online issue, which is available at wileyonlinelibrary.com.]

supplementation at day 7 resulted in lower blood glucose and elevated blood $\beta \mathrm{HB}$ in CR- and KE-treated animals compared to controls $\left({ }^{*} p<0.05, \quad{ }^{* *} p<0.01, \quad{ }^{* * *} p<0.001\right.$; one-way ANOVA; Figs. $4 d$ and $4 e$ ). By day 14, CR- and KE-treated mice lost $\sim 20 \%$ of their initial body weight $(p<0.01$; one-way ANOVA; Fig. 4f) and maintained that weight loss for the duration of the study. As expected, blood glucose and $\beta \mathrm{HB}$ were highly variable over the course of the survival study, likely owing to ad libitum feeding, health deterioration and cancerassociated cachexia (Figs. $4 g$ and $4 h$ ). Day 7 blood glucose and body weight change were significantly correlated to survival $(p=0.0065$ and $p=0.0046$, respectively; linear regression analysis; Fig. 5). Interestingly, linear regression analysis of tumor bioluminescence versus blood $\beta \mathrm{HB}$ did not reveal a significant correlation.

\section{Discussion}

The Warburg effect is the most ubiquitous cancer phenotype, exhibited by most if not all cancer types. ${ }^{1}$ Exploiting the metabolic deficiencies of cancer cells should be prioritized, because this therapeutic strategy would likely prove effective against most cancers. Mitochondrial dysfunction underlies many aspects of cancer metabolic deficiency and prevents cancer cells from effectively using ketone bodies for energy. ${ }^{12,20}$ In our study, ketone supplementation decreased VM-M3 cell proliferation and viability (Fig. 1), confirming similar results demonstrated in other cancer types in vitro. ${ }^{6,11,12}$ Therefore, we hypothesized that dietary administration of ketone body precursors would inhibit disease progression in vivo. Indeed, dietary administration of ketone precursors, $\mathrm{BD}$ and $\mathrm{KE}$, increased mean survival time by 51 and $69 \%$, respectively, in VM-M3 mice with metastatic cancer (Fig. 3). These data support the use of supplemental ketone administration as a feasible and efficacious cancer therapy, which should be further investigated in additional animal models to determine its potential for clinical use.

Ketone supplementation decreased blood glucose after acute administration, decreased body weight with chronic adminis- tration and sustained ketosis in vivo, even when administered with a high-carbohydrate rodent chow in both healthy (VM/ Dk) and cancer (VM-M3) mice (Fig. 4). Our study demonstrates the ability of dietary administration of $\mathrm{BD}$ and $\mathrm{KE}$ to significantly elevate ketone bodies in vivo for at least $12 \mathrm{hr}$ in healthy VM/Dk mice and 7 days in VM-M3 cancer mice (Fig. 4). VM-M3 cancer is very aggressive, and health of control animals declines rapidly after tumor cell inoculation. With onset of disease progression, animal feeding behavior, body weight and blood metabolites vary considerably. Blood and body weight data before the onset of significant disease progression in all treatment groups (Figs. $4 d-4 f$ ) demonstrate the effects of the dietary therapies on blood metabolites in VM-M3 cancer mice largely aside from the confounding effects of disease progression. Figures $4 g$ and $4 h$ show blood glucose and $\beta \mathrm{HB}$ levels of VM-M3 mice over the course of the survival study. Disease progression altered feeding behavior, and animals often exhibited low blood glucose and elevated blood ketones before euthanasia. For example, nearly all CR mice were euthanized in the fifth week of the study, with their declining health reflected by the marked increase in blood ketones at week 4 (Fig. $4 h$ ).

It is important to note that the metabolic changes associated with acute and chronic ketosis are vast and can dramatically affect blood metabolite concentrations. In previous studies, chronic $\mathrm{BD}$ and $\beta \mathrm{HB}$ administration has been shown to decrease food intake in the rat and pigmy goat. ${ }^{39,40}$ Similarly, Veech and coworkers demonstrated that feeding a KEsupplemented diet increased malonyl-CoA, an anorexigenic metabolite known to decrease food intake. ${ }^{41}$ Ketone-induced appetite suppression may account for the decreased blood glucose and body weight seen in treated VM-M3 cancer mice (Fig. 4f). Additionally, prior studies suggest that ketones increase insulin sensitivity, ${ }^{41,42}$ which may be contributing to the decreased circulating blood glucose in KE-fed mice. Blood metabolite levels are dynamic and change rapidly with food consumption and tissue utilization. Because the VM-M3 cancer mice were fed ad libitum, blood glucose and ketone levels observed at the time of measurement during chronic supplementation were likely affected by variable feeding behavior 
before blood measurement. As mentioned, health status of the animals also altered feeding behavior. Mice reduced food intake and likely experienced cancer-associated cachexia with disease progression, conditions that elevate blood ketones endogenously through beta-oxidation of stored fats. Furthermore, chronic ketosis enhances ketone utilization by tissues, known as keto-adaptation, resulting in lower blood ketone concentrations. ${ }^{43}$ All these factors likely contributed to the observed variability in blood glucose and $\beta \mathrm{HB}$ between acute versus chronic administration of diet and healthy $\mathrm{VM} / \mathrm{Dk}$ versus VM-M3 cancer mice (Fig. 4).

Previously, dietary intervention in cancer has largely focused on carbohydrate restriction or CR to exploit the Warburg effect. CR has been shown to slow disease progression in a variety of cancer models. ${ }^{2,36,44-46}$ As described, dietary-induced ketosis often leads to reduced appetite, decreased calorie consumption and decreased body weight, creating the possibility that the effects of ketone supplementation could be indirectly due to $\mathrm{CR} .{ }^{20}$ Interestingly, although CR decreased blood glucose and elevated blood ketones, CR mice exhibited a trend of increased latency to disease progression and increased survival that was not statistically significant from controls in our study (Figs. 2 and 3). As described, some data suggest that elevated ketones are responsible for much of the anticancer efficacy of the ketogenic diet. ${ }^{10}$ Perhaps elevating ketones with exogenous sources such as ketone supplementation or a ketogenic diet, rather than elevating ketones endogenously through lipolysis such as occurs with $\mathrm{CR}$, provides a more effective anticancer strategy. Additionally, ketone supplementation may preserve lean muscle mass to a greater degree than $\mathrm{CR}$, and may therefore support overall health of the organism in this way. Because CR mice demonstrated a trend of increased latency to disease progression and survival time, and body weight change was correlated to survival (Fig. 5), indirect CR likely contributed to the efficacy of ketone supplementation but does not account for the significant increase in survival time in ketone-fed mice. Additionally, although $\mathrm{BD}$ increased mean survival time by $51 \%$, blood glucose was not significantly lower than in control animals, suggesting that decreased glucose availability was not the primary mechanism of efficacy (Figs. 3 and 4). KE treatment increased mean survival to a greater extent than $\mathrm{BD}(69 \%$ increase from control; Fig. 3). Notably, blood glucose and body weight were more decreased and ketones were more elevated in KEtreated animals, possibly owing to indirect $\mathrm{CR}$, and perhaps account for the enhanced efficacy of $\mathrm{KE}$ compared to $\mathrm{BD}$ treatment (Fig. 4). In vitro, $5 \mathrm{mM} \beta \mathrm{HB}$ supplementation decreased proliferation rate and viability of VM-M3 cells even when added to $25 \mathrm{mM}$ glucose media (Fig. 1). Indeed, ketone supplementation was as effective as glucose-restriction (low, $3 \mathrm{mM}$ glu) treatment in decreasing VM-M3 cell proliferation and viability (Fig. 1). These data support the in vitro and in vivo conclusions of Fine et al. suggesting that ketone bodies can inhibit cancer progression independently of other factors such as carbohydrate restriction or CR. ${ }^{6,10-12}$

Lisanti and coworkers have published reports that tumorassociated fibroblasts produce ketone bodies to fuel nearby cancer cells. ${ }^{47,48}$ In these reports, the authors coculture immortalized fibroblasts genetically altered to overexpress rate-limiting ketone production enzymes with breast cancer cells genetically altered to overexpress ketone utilization enzymes. Although this phenomenon may occur in the system created by the authors, there is no evidence that this situation would occur naturally in vivo. As described, the literature as a whole strongly suggests that cancer cells of various tissue types are unable to effectively use ketone bodies for fuel. Many cancers do not express the succinyl CoA 3-ketoacid CoA transferase (SCOT) enzyme that is necessary for ketone body utilization, ${ }^{49}$ while several reports have demonstrated a notable deficiency of cancer cell ketone metabolism in vitro. ${ }^{12,21}$ It is also widely accepted that ketone production occurs nearly exclusively in the liver, and there is no known metabolic pathway by which fibroblasts could produce ketones from glucose. Without evidence to support this phenomenon in a natural cellular environment, we continue to accept the notion that most cancer cells cannot effectively metabolize ketones for energy.

The ketogenic diet has been shown to enhance the efficacy of both radiation and chemotherapy in vivo. ${ }^{4,50,51}$ As supplemental ketones mimic the physiological ketosis induced by the ketogenic diet, combining supplemental ketone therapy with standard of care could produce similar effects, even if administered with a SD. Furthermore, the neuroprotective effects of ketone metabolism have been widely documented. ${ }^{52}$ Ketone metabolism protects normal cells from oxidative damage by decreasing mitochondrial ROS production and enhancing endogenous antioxidant defenses. ${ }^{13}$ Radiation and chemotherapy work in large part by inducing ROS production in the tumor, but simultaneously incur damage to normal tissue. Ketone metabolism by healthy tissue would likely mitigate some of the adverse side effects of standard of care as ketones have been shown to protect against oxidative stress. ${ }^{13,34}$ Our data strongly suggest that supplemental ketone administration could provide a safe, feasible and cost-effective adjuvant to standard care that should be further investigated in preclinical and clinical settings.

\section{References}

1. Seyfried TN, Shelton LM. Cancer as a metabolic disease. Nutr Metab (Lond) 2010;7:7.

2. Shelton LM, Huysentruyt LC, Mukherjee P, et al. Calorie restriction as an anti-invasive therapy for malignant brain cancer in the VM mouse. ASN Neuro 2010;2:e00038.

3. Mavropoulos J, Buschemeyer W, Tewari A, et al. The effects of varying dietary carbohydrate and fat content on survival in a murine LNCaP prostate cancer xenograft model. Cancer Prev Res (Phil) 2009;2: 557-65. 
4. Zuccoli G, Marcello N, Pisanello A, et al. Metabolic management of glioblastoma multiforme using standard therapy together with a restricted ketogenic diet: case Report. Nutr Metab (Lond) 2010;7:33.

5. Nebeling LC, Miraldi F, Shurin SB, et al. Effects of a ketogenic diet on tumor metabolism and nutritional status in pediatric oncology patients: two case reports. J Am Coll Nutr 1995; 14:202-8.

6. Magee BA, Potezny N, Rofe AM, et al. The inhibition of malignant cell growth by ketone bodies. Aust J Exp Biol Med Sci 1979;57:529-39.

7. Wheatley KE, Williams EA, Smith NC, et al. Low-carbohydrate diet versus caloric restriction: effects on weight loss, hormones, and colon tumor growth in obese mice. Nutr Cancer 2008; 60:61-8.

8. Rossifanelli F, Franchi F, Mulieri M, et al. Effect of energy substrate manipulation on tumor-cell proliferation in parenterally fed cancer-patients. Clin Nutr 1991;10:228-32.

9. Poff AM, Ari C, Seyfried TN, et al. The ketogenic diet and hyperbaric oxygen therapy prolong survival in mice with systemic metastatic cancer. PLoS One 2013;8:e65522.

10. Fine E, Segal-Isaacson C, Feinman R, et al. Targeting insulin inhibition as a metabolic therapy in advanced cancer: a pilot safety and feasibility dietary trial in 10 patients. Nutrition 2012;28: 1028-35.

11. Fine E, Miller A, Quadros E, et al. Acetoacetate reduces growth and ATP concentration in cancer cell lines which over-express uncoupling protein 2. Cancer Cell Int 2009;9:14

12. Skinner R, Trujillo A, Ma X, et al. Ketone bodies inhibit the viability of human neuroblastoma cells. J Pediatr Surg 2009;44:212.

13. Veech RL, Chance B, Kashiwaya Y, et al. Ketone bodies, potential therapeutic uses. IUBMB Life 2001;51:241-7.

14. Isidoro A, Martínez M, Fernández $\mathrm{P}$, et al. Alteration of the bioenergetic phenotype of mitochondria is a hallmark of breast, gastric, lung and oesophageal cancer. Biochem J 2004;378 (Part 1): $17-20$.

15. Kiebish MA, Han X, Cheng H, et al. Brain mitochondrial lipid abnormalities in mice susceptible to spontaneous gliomas. Lipids 2008;43:951-9.

16. Kiebish MA, Han X, Seyfried TN. Examination of the brain mitochondrial lipidome using shotgun lipidomics. Methods Mol Biol 2009;579: 3-18.

17. Arismendi-Morillo G, Castellano-Ramirez A. Ultrastructural mitochondrial pathology in human astrocytic tumors: potentials implications pro-therapeutics strategies. J Electron Microsc 2008;57:33-42.

18. Kiebish MA, Han X, Cheng H, et al. Cardiolipin and electron transport chain abnormalities in mouse brain tumor mitochondria: lipidomic evidence supporting the Warburg theory of cancer. J Lipid Res 2008;49:2545-56.

19. Modica-Napolitano J, Singh K. Mitochondrial dysfunction in cancer. Mitochondrion 2004;4: $755-817$.
20. Seyfried TN. Cancer as a metabolic disease: on the origin, management, and prevention of cancer. Hoboken, NJ: Wiley, 2012.

21. Maurer G, Brucker D, Bähr O, et al. Differential utilization of ketone bodies by neurons and glioma cell lines: a rationale for ketogenic diet as experimental glioma therapy. BMC Cancer 2011, 11:315.

22. Wu GY, Thompson JR. The effect of ketone bodies on alanine and glutamine metabolism in isolated skeletal muscle from the fasted chick. Biochem J 1988;255:139-44.

23. Schumacker P. Reactive oxygen species in cancer cells: live by the sword, die by the sword. Cancer Cell 2006;10:175-81

24. Dhup S, Dadhich RK, Porporato PE, et al. Multiple biological activities of lactic acid in cancer: influences on tumor growth, angiogenesis and metastasis. Curr Pharm Des 2012;18:1319-30.

25. Shimazu T, Hirschey M, Newman J, et al. Suppression of oxidative stress by $\beta$-hydroxybutyrate, an endogenous histone deacetylase inhibitor. Science 2013;339:211-14.

26. Ropero S, Esteller M. The role of histone deacetylases (HDACs) in human cancer. Mol Oncol 2007;1:19-25.

27. Gupta G, Massagué J. Cancer metastasis: building a framework. Cell 2006;127:679-95.

28. Huysentruyt LC, Mukherjee P, Banerjee D, et al. Metastatic cancer cells with macrophage properties: evidence from a new murine tumor model. Int J Cancer 2008;123:73-84.

29. Shelton LM, Mukherjee P, Huysentruyt LC, et al. A novel pre-clinical in vivo mouse model for malignant brain tumor growth and invasion. J Neurooncol 2010;99:165-76.

30. Huysentruyt L, Akgoc Z, Seyfried T. Hypothesis: are neoplastic macrophages/microglia present in glioblastoma multiforme? ASN Neuro 2011;3: 183-93.

31. Huysentruyt LC, Shelton LM, Seyfried TN. Influence of methotrexate and cisplatin on tumor progression and survival in the VM mouse model of systemic metastatic cancer. Int J Cancer 2010;126: 65-72.

32. Kies C, Tobin RB, Fox HM, et al. Utilization of 1,3-butanediol and nonspecific nitrogen in human adults. J Nutr 1973;103:1155-63.

33. Mehlman M, Veech R. Redox and phosphorylation states and metabolite concentrations in frozen clamped livers of rats fed diets containing 1,3-butanediol and DL-carnitine. J Nutr 1972;102: $45-51$.

34. D'Agostino DP, Pilla R, Held HE, et al. Therapeutic ketosis with ketone ester delays central nervous system oxygen toxicity seizures in rats. Am J Physiol Regul Integr Comp Physiol 2013;304: R829-R836.

35. Rossi R, Dorig S, Del Prete E, et al. Suppression of feed intake after parenteral administration of D-beta-hydroxybutyrate in pygmy goats. $J$ Vet Med A Physiol Pathol Clin Med 2000;47:9-16.

36. Hursting S, Smith S, Lashinger L, et al. Calories and carcinogenesis: lessons learned from 30 years of calorie restriction research. Carcinogenesis 2010;31:83-9.
37. Lim E, Modi KD, Kim J. In vivo bioluminescent imaging of mammary tumors using IVIS spectrum. J Vis Exp 2009;26:e1210, doi: 10.3791/1210.

38. Lim E, Modi K, Christensen A, Meganck J, Oldfield S, Zhang N. Monitoring tumor metastases and osteolytic lesions with bioluminescence and micro CT imaging. J Vis Exp 2011; 50. http://www.jove.com/details.php?id=2775, doi: 10.3791/2775

39. Arase K, Fisler JS, Shargill NS, et al. Intracerebroventricular infusions of $3-\mathrm{OHB}$ and insulin in a rat model of dietary obesity. Am J Physiol 1988; 255(6 Part 2):R974-R981.

40. Carpenter RG, Grossman SP. Plasma fat metabolites and hunger. Physiol Behav 1983;30: 57-63.

41. Kashiwaya Y, Pawlosky R, Markis W, et al. A ketone ester diet increases brain malonyl-CoA and Uncoupling proteins 4 and 5 while decreasing food intake in the normal Wistar Rat. $J$ Biol Chem 2010;285:25950-6.

42. Amiel SA, Archibald HR, Chusney G, et al. Ketone infusion lowers hormonal responses to hypoglycaemia: evidence for acute cerebral utilization of a non-glucose fuel. Clin Sci (Lond) 1991;81:189-94.

43. Westman EC, Feinman RD, Mavropoulos JC, et al. Low-carbohydrate nutrition and metabolism. Am J Clin Nutr 2007;86:276-84.

44. Berrigan D, Perkins S, Haines D, et al. Adultonset calorie restriction and fasting delay spontaneous tumorigenesis in p53-deficient mice. Carcinogenesis 2002;23:817-22.

45. Mukherjee P, Abate LE, Seyfried TN. Antiangiogenic and proapoptotic effects of dietary restriction on experimental mouse and human brain tumors. Clin Cancer Res 2004;10: 5622-9.

46. Seyfried B, Kiebish M, Marsh J, et al. Targeting energy metabolism in brain cancer through calorie restriction and the ketogenic diet. J Cancer Res Ther 2009; 5 (Suppl 1):15.

47. Martinez-Outschoorn UE, Lin Z, WhitakerMenezes D, et al. Ketone body utilization drives tumor growth and metastasis. Cell Cycle 2012;11: 3964-71.

48. Bonuccelli G, Tsirigos A, Whitaker-Menezes D, et al. Ketones and lactate "fuel" tumor growth and metastasis: evidence that epithelial cancer cells use oxidative mitochondrial metabolism. Cell Cycle 2010;9:3506-14.

49. Tisdale M, Brennan R. Loss of acetoacetate coenzyme A transferase activity in tumours of peripheral tissues. Br J Cancer 1983;47:293-7.

50. Marsh J, Mukherjee P, Seyfried T. Drug/diet synergy for managing malignant astrocytoma in mice: 2-deoxy-D-glucose and the restricted ketogenic diet. Nutr Metab (Lond) 2008;5:33.

51. Abdelwahab M, Fenton K, Preul M, et al. The ketogenic diet is an effective adjuvant to radiation therapy for the treatment of malignant glioma. PLoS One 2012;7:e36197.

52. Maalouf M, Rho J, Mattson M. The neuroprotective properties of calorie restriction, the ketogenic diet, and ketone bodies. Brain Res Rev 2009;59: 293-315. 